\section{Monitoring and anaesthetic management of parturients with mitral stenosis}

To the Editor:

I read with interest the article of Hemmings et al. ${ }^{1}$ concerning the intrapartum management of a patient with mitral stenosis. These authors noted several differences from our previous work. ${ }^{2}$ Haemodynamic observations differed from patients in our series in several respects. Most notably, Hemmings et al. did not observe a postpartum increase in pulmonary capillary wedge pressure. While they mentioned several factors which may account for this difference, principally the use of epidural anaesthesia, a difference in patient selection may also have played a role. The patient described by Hemmings es al. experienced the onset of dyspnoea on exertion after walking six blocks, by definition, New York Heart Association Class Functional II. In our series, only patients who were Functional Class III or IV were included. In this group of patients, the ability to tolerate the postpartum autotransfusion phenomenon might be expected to be less than a patient with less severe valvular disease who had previously undergone commissurotomy, confirming our observation that the postpartum rise in PCWP was most marked in those patients with the most severe valvular disease.

\section{Steven L. Clark MD}

Utah Valley Regional Perinatal Center

1034 North. 500 West

Provo, Utah

84604

U.S.A.

\section{REFERENCES}

1 Hemmings GT, Whalley $D G, O^{\prime}$ Connor PJ, Benjamin $A$, Dunn $C$. Invasive monitoring and anaesthetic management of a parturient with mitral stenosis. Can J Anaesth 1987; 34: 182-5.

2 Clark SL, Phelan JP, Greenspoon J, Aldaht D, Horenstein J. Labor and delivery in the presence of mitral stenosis: central hemodynamic observations. Am J Obstet Gynecol 1985; 152: 984.

\section{REPLY}

We appreciate Dr. Clark's interest in our report and would accept his suggestion that patients at a functional classification mare severe than the patient on whom we reported might respond differently to post-partum auto transfusion. As I am sure he will agree, we do not have the database from one patient to confirm this, but would suggest that the more severely functionally compromised parturient with mitral stenosis might especially benefit from the peripheral vasodilating effects of epidural anaesthesia maintained well into the post-partum period.

Based on our experience, we feel comfortable in recommending epidural anaesthesia for parturients for mitral stentosis provided switable haemodynamic monitoring is available.

\section{D.G. Whalley MB}

G.T. Hemmings MD, FRCPC

Department of Anacsthesia

Royal Victoria Hospital

and McGill University

Montreal, Quebec

H3A 1 A 1

\section{CPAP circuit for non-ventilated lung during thoracic surgery}

To the Editor:

We would like to describe a modified Mapleson D anaesthetic circuit that we have developed to provide continuous positive airway pressure (CPAP) to the nonventilated lung during thoracic surgery. The surgeon frequently needs the operative lung temporarily reinflated in mid-operation to check for air leaks in the bronchial suture lines. Technically this is very awkward using previously described CPAP circuits. ${ }^{1}, 2$

Our circuit (Figure) is composed of a $22 \mathrm{~mm}$ polyethylene corrugated tubing with oxygen side-arm connectors (U/adapit ${ }^{\circledR}$, Airlife Inc., Montclair, California) at each end. The oxygen inflow and outflow lines are standard $4 \mathrm{~mm}$ polyethylene tubing. At the distal end of the circuit is a "pop-off" valve (closed during CPAP) and a three litre reservoir bag. The oxygen outflow line exits at a variable distance underwater in an occluded $50 \mathrm{ml}$ syringe. We sccure the oxygen line in the syringe by passing it through the perforated plunger of a $20 \mathrm{ml}$ syringe which is then fitted into a "T" piece and wedged into the open end of the $50 \mathrm{ml}$ syringe. (Alternately the line can be passed through the plunger of the $50 \mathrm{ml}$ syringe and a side-hole opened near the top of the syringe.)

Temporary clamping of the oxygen outflow line permits inflation of the non-ventilated lung, by pressure on the reservoir bag, when required for surgical purposes. Releasing the clamp will allow the lung to deflate to its previous level of CPAP. The oxygen inflow line can be clamped to permit assessment of air leak from the non-ventilated lung to assure isolation of one lung from the other.

The level of CPAP in the circuit corresponds to the number of centimeters the distal orifice of the oxygen 


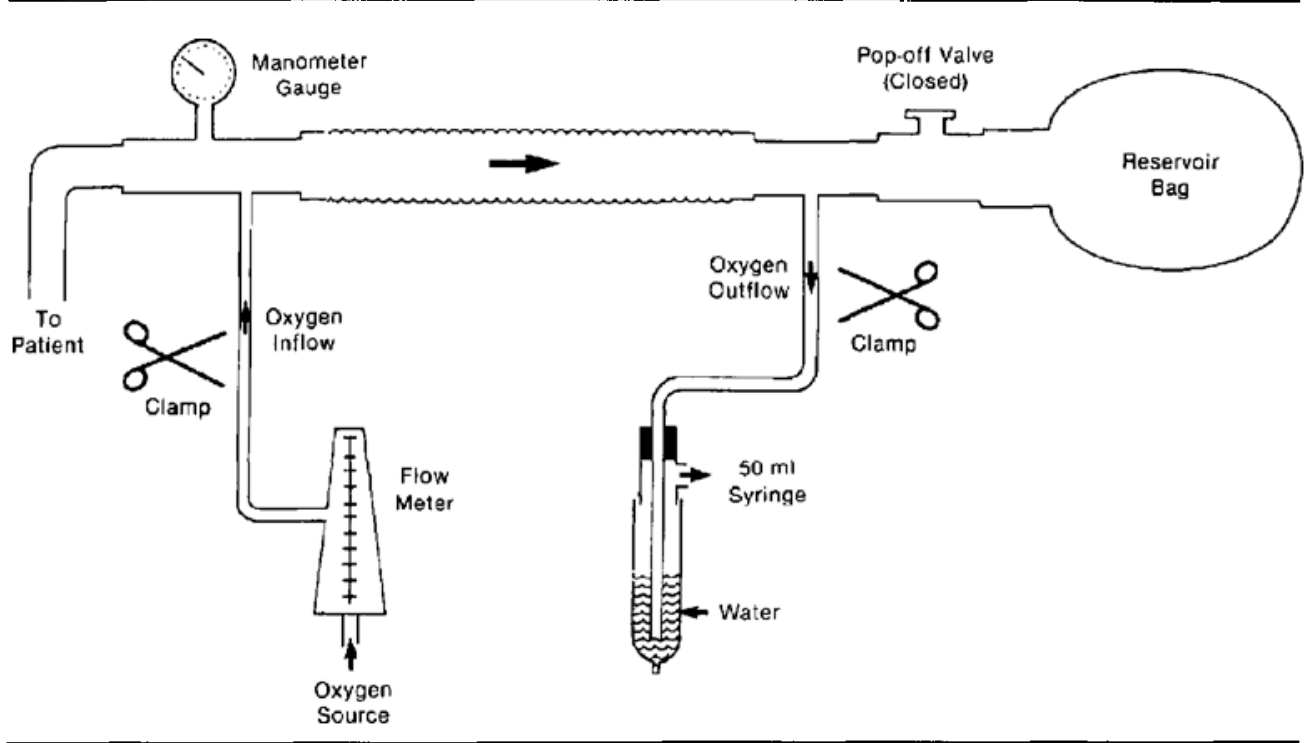

FIGURE Cireuit to provide CPAP to the non-ventilated lung during one-lung anaesthesia. The patient end is connected to the non-ventilated lumen of a double-lumen endobronchial tube.

outflow tubing lies below the surface of the water in the syringe. Individual requirements for CPAP may depend on individual lung mechanics. This circuit permits finetuning of the CPAP level by simply adjusting the depth the oxygen outflow lies underwater.

We usually use an oxygen inflow of $1.0 \mathrm{~L} \cdot \mathrm{min}^{-1}$. At flows less than $2.0 \mathrm{~L} \cdot \mathrm{min}^{-1}$ we find the manometer measured pressure in the circuit is the same as the level set by the depth of the underwater outflow line (at similar low oxygen flows we feel it is not strictly necessary to incorporate a manometer in the circuit).

The "pop-off" valve and reservoir bag can be autoclaved or chemically disinfected, the remainder of the circuit is disposable.

We have used this circuit to improve oxygenation in over 100 thoracotomies in the past two years without any problem related to its use. It combines the ability to ventilate with the safety and physical advantages of underwater seal pressure.

\section{P. Slinger, MD FRCPC}

W. Triolet, MD FRCPC

M. Chang, CSRT RRAT

Department of Anaesthesia

Montreal General Hospital

Montreal, Quebec

H3G 1 A4

\section{REFERENCES}

1 Baraka A, Sibai AN, Mullaem M, Baroody $M$, Haroun 5, Mekkaoui T. CPAP oxygenation during one-lung ventilation using an under-water seal assembly. Anesthesiology 1986; 65: 102-3.

2 Thiagarajah $S, J o b C, R a o A$. A device for applying CPAP to the non-ventilated upper lung during one-lung ventilation. Anesthesiology 1984; 60: 254-5.

\section{Respiratory complications in patients with upper respiratory tract infections}

\section{To the Editor:}

The conclusions drawn in the article by Tait and Knight ${ }^{1}$ must be viewed with skepticism.

This study was retrospective. Only patients having received a general anaesthetic were therefore included. For patients whose surgery was cancelled, the authors did not identify the criteria which were used for cancellation because of an upper respiratory infection. As a result, it is possible (and even likely) that patients who had the most obvious and serious symptoms of upper respiratory infection were not included. The conclusion that ithere is 